\title{
A comparison of the effects of medial and lateral midbrain lesions in rats'
}

\author{
ANNETTE EHRLICH, REGIONAL PRIMATE RESEARCH CENTER, UNIVERSITY OF WASHINGTON
}

STEPHEN E. GLICKMAN, UNIVERSITY OF MICHIGAN

In rats, both medial and lateral midbrain lesions produced a signific ant decrease in bar pressing for food. During a test session, animals with midbrain lesions made a greater number of active responses unrelated to food than did the controls. The motivational changes are ascribed to reticular damage.

A previous study (Ehrlich, 1963) showed that, in rats, small midbrain lesions decrease food motivation, as measured by bar pressing in a Skinner box, but leave other measures of hunger, such as ad lib food intake and weight gain, unaltered. The aims of the present study were twofold: first, to define more precisely the effective midbrain locus by comparing the effects of medial as opposed to lateral midbrain lesions and second, to determine, by means of a time sampling procedure, what sort of behavior occurs in animals that are notas motivated as normals to work for food.

\section{Method}

Ss were 34 female albino rats, divided into the following groups: medial-10, lateral -10 , sham operate -8 , and normal control-6. Apparatus consisted of three identical Skinner boxes. Bilateral midbrain lesions were made electrolytically in the two experimental groups. In the sham operate group the electrode was lowered but no current was passed. At the conclusion of the experiment the brains of all but the normal controls were subjected to histological examination. No damage was found in the sham operates. Figure 1 shows some reconstructions of typical lesions in the two midbrain groups.

During the bar-pressing test the animals were on a 24-hr. deprivation schedule. Tests lasted $20 \mathrm{~min}$. and each reinforced press delivered a $45-\mathrm{mg}$. food pellet. Each bar-pressing test was followed by a 20 -min. feeding period during which the animals were offered Purina Fox Chow meal mixed with water. The rats were trained preoperatively to bar press for food on a continuous reinforcement schedule. At the end of the five-day training period, a preoperative bar-pressing score was computed by averaging each animal's scores for days $2-5$. The animals were then divided into four groups with approximately equal means, and the operations were performed. A four-day retest was begun 21 days after the operations and the pre- and postoperative barpressing scores were compared. Subsequently, each animal was given a single 5-min. bar-pressing test during which a record was kept of what the animal was doing at the beginning of each 6-sec.interval. A total of 50 responses was recorded for each rat and the following categories were used: (1) Responses related to food-

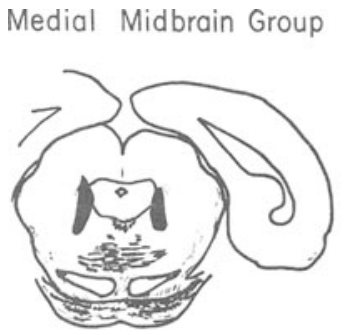

Lateral Midbrain Group
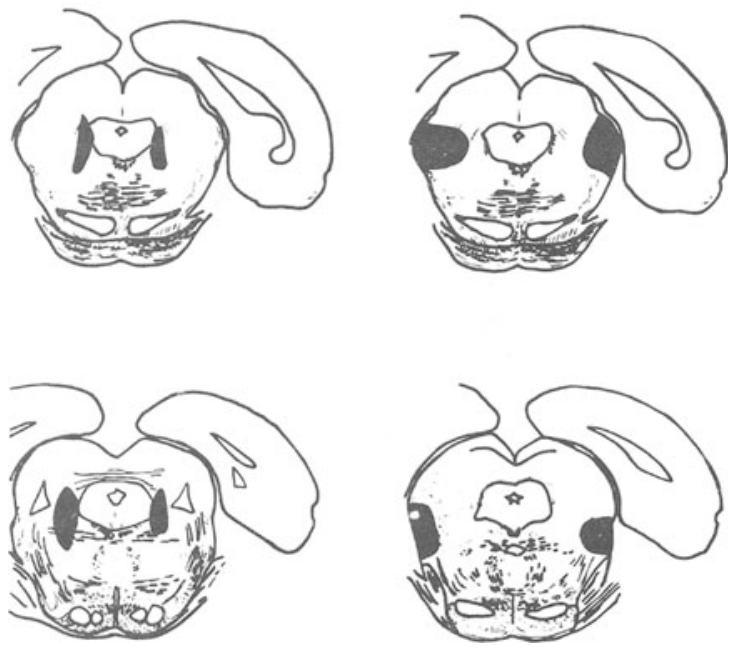

Fig. 1. Reconstructions of typical lesions in the lateral and medial midbrain groups.

bar pressing, sniffing, licking, or chewing bar, chewing pellets. (2) Active responses unrelated to food-rearing, walking, grooming, sniffing. (3) Passive responses unrelated to food-sitting, lying. (4) Miscellaneous responses-drinking; anything not included in the above categories.

\section{Results}

Table 1 shows the mean pre- and postoperative barpressing scores, as well as the mean difference between these scores, for each group. Analysis of variance of these difference scores showed that the overall variance between groups was significant $(F=15.5, p<.01)$. Comparisons between individual group means were then made by means of $t$ tests, and the results were as follows: (1) The difference between the two groups with midbrain lesions was not significant $(t=.53, p>.20)$ 。 (2) The operations significantly lowered the bar-

Table 1. Effect of Operation on Bar Pressing for Food On a Continuous Reinforcement Schedule

$$
\text { Presses Per 20-min. Session }
$$

Group Preoperative Mean Postoperative Mean Mean Differences

\begin{tabular}{llrr} 
Normal control & 135.1 & 164.9 & 29.3 \\
Sham operate & 125.9 & 99.8 & -26.1 \\
Medial midbrain & 145.5 & 39.3 & -56.2 \\
Lateral midbrain & 142.2 & 78.8 & -63.4 \\
\hline
\end{tabular}


pressing rate of the two midbrain-lesion groups by comparison with both the normal controls $(t=5.91$, $\mathrm{p}<.001$ for the normal and lateral midbrain groups; $t=5.45, p<.001$ for the normal and medial midbrain groups) and the sham operate group $(t=2.57, p<.02$ for the sham operate and lateral midbrain groups; $t=2.07$, $\mathrm{p}<.05$ for the sham operate and medial midbrain groups). (3) Sham operations also produced a decline in bar pressing. Although the effect was notas great as that seen in the two midiorain-lesion groups, the sham operate group declined significantly by comparison with the normal control group $(t=3.39, p<.01)$.

Of the 50 responses sampled during bar pressing, the distribution among the various categories was quite consistent from group to group and, as one would expect, the most frequently sampled responses were those related to food. Separate analyses of variance carried out for each of the four response categories revealed significant between-group variances for only two categoriesnumber of responses related to food $(F=5.42, p<.01)$ and number of active responses unrelated to food $(F=3.91, p<.05)$. Separate comparisons revealed that there were no significant differences between the two control groups or between the two lesion groups in either of these two categories, and the data were therefore pooled so that comparisons could be made between the combined control group and the combined operate group. In the category of food-related responses, the controls made significantly more responses than the operate animals (means = 43.82 and 35.89 for controls and for operates respectively; $t=3.84, p<.008$ ). In the category of active responses unrelated to food, the operate animals made significantly more responses than the controls (means $=\mathbf{1 0 . 5 5}$ and $\mathbf{5 . 2 6}$ for the operates and controls respectively).

Discussion

The results reported here confirm and extend the earlier findings (Ehrlich, 1963). Both medial and lateral midbrain lesions produce a significant deficit in bar pressing for food; further, the effect is not limited to a single sex or strain, for it occurs in female albinos (used in the present experiment) as well as in male hooded rats (used in the previous study). The earlier report showed that the motivational change was not due to any alteration in ad lib food intake, and the time sampling data reported here rule out another possible explanation, based on somnolence, for when they were not bar pressing or eating, operates tended to be active rather than passive.

One puzzling feature of the present results is the significant deficit found in the sham operate group. While the reason for this finding is not apparent, it is clear that the lesions had an additional effect since the performance of the two groups with midbrain lesions was significantly below that of the sham operate group. Further, that the deficit found in the two midbrainlesion groups is specific to lesions in that part of the brain is shown by the finding in an earlier study that control lesions in the area of the fornix and hippocampal commissure produce a significant increase rather than a decrease in bar pressing for food (Ehrlich, 1963).

Because the midbrain lesions involved both specific and nonspecific systems it is difficult to correlate the behavioral change with destruction of some precise focus. However, several factors suggest that damage to nonspecific pathways was the crucial factor. First, both the lateral and the medial lesions spared the main ascending sensory pathways in and around the medial lemniscus. Second, although specific pathways were hit in both lesion groups, the damage was largely confined to auditory structures (lateral lemniscus and inferior colliculus) in the lateral group whereas these structures were spared in the medial group. Third, the main point of overlap between the two types of lesion is that both involved some reticular damage.

\section{Reference}

Ehrlich, Annette. Effects of tegmental lesions on motivated behavior in rats. J. comp. physiol. Psychol., 1963, 56, 390-396.

\section{Note}

1. This research was supported by grant number 1771 from the Illinois State Psychiatric Research and Training Authority and by grant number G179496 from the National Science Foundation and was carried out while the first author was at Chicago Wesley Memorial Hospital and the second author was at Northwestern University. 